\title{
Tryptophan-based chromophore in fluorescent proteins can be anionic
}

\author{
SUBJECT AREAS: \\ MOLECULAR \\ ENGINEERING \\ IMAGING \\ PROTEINS \\ PROTEIN DESIGN \\ Karen S. Sarkisyan ${ }^{1,3}$, llia V. Yampolsky' , Kyril M. Solntsev², Sergey A. Lukyanov ${ }^{1,3}$, \\ Konstantin A. Lukyanov' \& Alexander S. Mishin ${ }^{1,3}$

\begin{abstract}
${ }^{1}$ Shemyakin-Ovchinnikov Institute of Bioorganic Chemistry, Moscow, Russia, ${ }^{2}$ School of Chemistry and Biochemistry, Georgia Institute of Technology, 901 Atlantic Drive, Atlanta, Georgia 30332-0400, United States, ${ }^{3}$ Nizhny Novgorod State Medical Academy, Nizhny Novgorod, Russia.
\end{abstract}

Received

1 May 2012

Accepted

31 July 2012

Published

29 August 2012

Correspondence and requests for materials should be addressed to A.S.M. (mishin@ibch. ru)
Cyan fluorescent proteins (CFP) with tryptophan66-based chromophore are widely used for live cell imaging. In contrast to green and red fluorescent proteins, no charged states of the CFP chromophore have been described. Here, we studied synthetic CFP chromophore and found that its indole group can be deprotonated rather easily ( $\mathrm{pKa} 12.4)$. We then reproduced this effect in the CFP mCerulean by placing basic amino acids in the chromophore microenvironment. As a result, green-emitting variant with an anionic chromophore and key substitution Val61Lys was obtained. This is the first evidence strongly suggesting that tryptophan-based chromophores in fluorescent proteins can exist in an anionic charged state. Switching between protonated and deprotonated Trp66 in fluorescent proteins represents a new unexplored way to control their spectral properties.

luorescent proteins (FP) of GFP family represent a powerful tool to visualize structures and dynamic
processes in live systems ${ }^{1}$. FPs carry chromophores formed by self-catalyzed modifications of three internal
amino acids (e.g., Ser65-Tyr66-Gly67 in GFP from jellyfish Aequorea victoria). All known natural FPs of different colors have Tyr66 as a central chromophore-forming residue. A very important feature of chromophore's Tyr66 is its ability to exist either in neutral (protonated) or anionic (deprotonated) states, depending on $\mathrm{H}$-bond network around phenolic oxygen ${ }^{1}$. Corresponding neutral and anionic chromophores possess drastically different spectral properties. Anionic GFP-like chromophore absorbs blue light at about $480 \mathrm{~nm}$ and emits green light at about $510 \mathrm{~nm}$. In contrast, neutral GFP-like chromophore absorbs violet light at about $400 \mathrm{~nm}$ and can either emit blue light at about $450 \mathrm{~nm}$, or, via excited state proton transfer mechanism it can undergo ultrafast conversion into anionic state with green emission at about $510 \mathrm{~nm}$. Similar behavior was also described for red fluorescent proteins ${ }^{2}$. Interconversions between neutral and anionic states in fluorescent proteins with Tyr66based chromophores represent a structural basis for functioning of a number of fluorescent sensors, as well as photoactivatable fluorescent proteins, which are widely used for protein, organell and cell tracking and superresolution imaging ${ }^{1}$.

While natural FPs have Tyr66-based chromophores, this residue can be artificially substituted for other aromatic amino acids resulting in blue-shifted variants - cyan FPs (CFP) with Trp66 and blue FPs with His66 or Phe66 ${ }^{3}$. CFPs appeared to be a particularly useful for multicolor labeling and FRET-based applications. Recently, greatly improved variants, namely Cerulean, Cerulean3, mTurquoise and mTurquoise 2 were gener$\operatorname{ated}^{4-7}$.

Up to date, no charged states of the Trp66-based CFP chromophore were described. Even theoretical possibility of existence of such variants was not discussed. Here, we describe the first fluorescent protein with anionic Trpbased chromophore obtained by directed molecular evolution of Cerulean.

\section{Results}

Tryptophan side chain does not exist in anionic state in biological systems because of extremely high $\mathrm{p} K_{\mathrm{a}}$ (about 20) of indole deprotonation. However, in the case of extended aromatic system of Trp-based chromophore some changes in $\mathrm{p} K_{\mathrm{a}}$ might be expected due to more efficient delocalization of negative charge. Indeed, we noticed that under strong basic conditions synthetic CFP chromophore ${ }^{8}$ ((5Z)-5-(1H-indol-3-ylmethylidene)-2,3-dimethyl3,5-dihydro-4H-imidazol-4-one) undergoes bathochromic shift of about $60 \mathrm{~nm}$ with $\mathrm{p} K_{\mathrm{a}} 12.4$ (Supplementary Fig. 1). We observed a similar bathochromic shift in the absorption spectrum of cyan fluorescent protein mCerulean upon denaturation in $5 \mathrm{M} \mathrm{NaOH}$. The most likely explanation of such a change in the spectrum is 

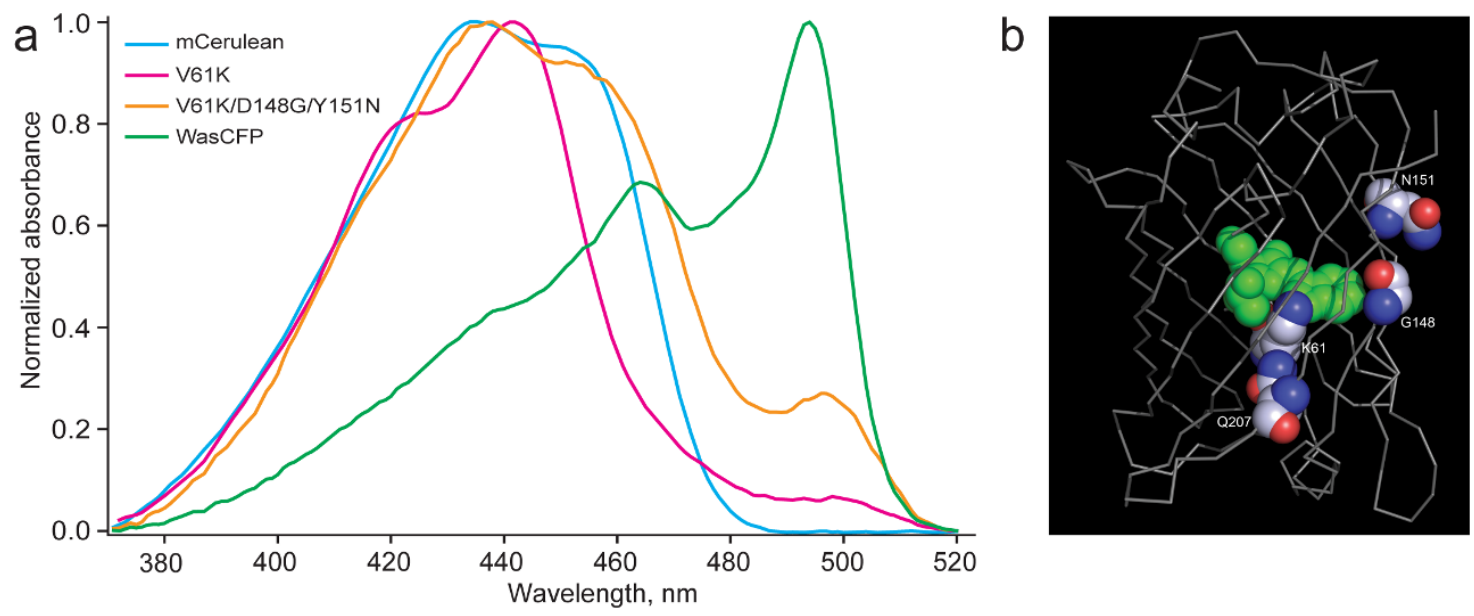

Figure 1 Site-directed and random mutagenesis of mCerulean. (a) Normalized absorption spectra of mCerulean and its mutants collected from sequential rounds of mutagenesis. All spectra were measured at $\mathrm{pH} 7.4$ and $4{ }^{\circ} \mathrm{C}$. Black line - Cerulean, blue - V61K, cyan - V61K/D148G/Y151N, green - WasCFP (V61K/D148G/Y151N/L207Q) mutants. (b) Modeling of mutations V61K, D148G, Y151N and L207Q on Cerulean crystal structure (PDB ID: 2WSO) using PyMol package.

the deprotonation of indole nitrogen. These results encouraged us to perform mutagenesis aimed to generate a fluorescent protein with anionic Trp66-based chromophore. We expected spectra of the anionic state of Trp-based chromophore to be red-shifted as compared to the neutral state.

Using cyan FP mCerulean as a template we introduced Lys and Arg residues at positions nearby the Trp66, specifically, positions 61, 146,203 , and 205 . We assumed that positively charged side chains of these amino acids could potentially stabilize a negative charge on the indole group. Indeed, one variant carrying substitution V61K demonstrated a minor but clearly detectable additional red-shifted fluorescence excitation peak at $494 \mathrm{~nm}$ with emission at $505 \mathrm{~nm}$ (not shown). Absorption spectrum of mCerulean-V61K suggested that this new spectral feature corresponded to a small fraction of the bulk protein (Fig. 1). Therefore, random mutagenesis was used to enhance the relative intensity of green fluorescence, as well as overall brightness. Variants selected on sequential rounds of random mutagenesis showed gradually increasing fraction of 494-nm absorption peak (Fig. 1). Final variant, named WasCFP (W in anionic state CFP), carried amino acid substitutions V61K, D148G, Y151N, and L207Q and demonstrated mainly green fluorescence (excitation and emission maxima at 494 and $505 \mathrm{~nm}$, respectively, quantum yield 0.85; extinction coefficient at $494 \mathrm{~nm} 51000 \mathrm{M}^{-1} \mathrm{~cm}^{-1}$ ), although the Cerulean-like cyan component (excitation and emission maxima at 437 and $477 \mathrm{~nm}$, respectively, quantum yield 0.48 ; extinction coefficient at $437 \mathrm{~nm} 28000 \mathrm{M}^{-1} \mathrm{~cm}^{-1}$ ) remained as well.

$\mathrm{pH}$-dependent spectral changes in fluorescent proteins spectra are routinely used to determine chromophore protonation state ${ }^{1-3,9}$. In particular, one could expect increase in partial content of anionic form of the chromophore with increase in $\mathrm{pH}$. WasCFP spectra possessed strong $\mathrm{pH}$ and temperature dependences with fast and reversible interconversion between the cyan and green forms (Fig. 2, Supplementary Fig. 2a,b, Supplementary Fig. 3). Alkaline $\mathrm{pHs}$ and/or low temperatures stabilized the green form of WasCFP. For example, a dominated green form can be obtained at $\mathrm{pH} 7.4$ and $4{ }^{\circ} \mathrm{C}$, or at $\mathrm{pH} 8.1$ and $25^{\circ} \mathrm{C}$. Notably, the absorption spectrum of WasCFP green form possessed a red shift similar to that of mCerulean denatured in strong alkaline conditions where its chromophore becomes deprotonated (Fig. 2a). We concluded that green form of WasCFP carries chromophore with Trp66 in anionic state (Fig. 2c).

At high $\mathrm{pH}$ values a drop in absorption at $494 \mathrm{~nm}$ occurred (Supplementary Fig. 2a,b). This transition with apparent $\mathrm{p} K_{\mathrm{a}}$ of about 9.8 can be explained by titration of a lysine side chain (including
Lys61) or overall change of protein conformation and hydrogen bond network around the chromophore in alkali.

WasCFP exhibited an unusual spectral behavior in urea solutions. Typically, fluorescent proteins are resistant to denaturants such as $8 \mathrm{M}$ urea or $6 \mathrm{M}$ guanidine hydrochloride ${ }^{9,10}$. In contrast, WasCFP absorption changed in the presence of as low as $0.25 \mathrm{M}$ urea (Supplementary Fig. 2c). Interestingly, presence of urea resulted in increase of 494-nm peak (green form), but did not affect absorption at shorter wavelengths (cyan form).

To gain a better understanding of this multicomponent absorption-emission system we have performed a full 3D excitation/emission scan of WasCFP at various $\mathrm{pH}$ (Fig. 3). At all $\mathrm{pH}$ values in the range of 6.4-9.4 two very closely lying emission centers were observed: the weakly emissive component with excitation/emission at about $450 / 475 \mathrm{~nm}$, and the dominating component with excitation/emission at about 495/505 $\mathrm{nm}$. As mentioned before, we assigned them to the cyan and the green emitting forms, respectively. Spectral analysis of the $2 \mathrm{D}$ contour plots demonstrated that at all $\mathrm{pH}$ the green component has the same excitation spectra, therefore excluding the possibility of the cyan to green conversion via excited state proton transfer (ESPT).

We have also measured the 3D fluorescence decay surfaces of the abovementioned samples upon $372 \mathrm{~nm}$ and $467 \mathrm{~nm}$ pulsed laser excitation (Fig. 4). The $372 \mathrm{~nm}$ excitation would lead to simultaneous excitation of the cyan and green forms, while the $467 \mathrm{~nm}$ excitation would predominantly excite the green form. Indeed, the $372 \mathrm{~nm}$ excitation led to mutliexponential decays at all wavelengths throughout the emission spectrum. The individual decays at various wavelengths reflected the overlapped emission of the cyan and the green bands. However, we have noticed that the decays measured in the red part of the spectrum were longer than those for the blue part. Some selected decay curves monitored at $510 \mathrm{~nm}$ are presented in Fig. $4 \mathrm{~b}$. To reveal the origin of the decaying species we have performed the global analysis of the kinetic data (excitation $372 \mathrm{~nm}, \mathrm{pH} 7.4$ ). As expected, the decay-associated species (DAS) were grouped around $490 \mathrm{~nm}$ and $515 \mathrm{~nm}$ (Supplementary Fig. 4). The $490 \mathrm{~nm}$ species can be best characterized with 3 decaying components with the lifetimes of $55 \mathrm{ps}, 505 \mathrm{ps}$, and $2.2 \mathrm{~ns}$, while the $515 \mathrm{~nm}$ band has the unusually long $6.0 \mathrm{~ns}$ decay. In all cases no detectable risetimes were detected at all wavelengths, ruling out the ESPT mechanism.

Upon $467 \mathrm{~nm}$ excitation of all samples, no time evolution of the emission spectra was detected, and the decay of the green species was exponential with the lifetime of 5.2 ns at $\mathrm{pH} 7.0$, and 5.0 at $\mathrm{pH} 9.4$. We think that these values are more reliable than the ones obtained 
a

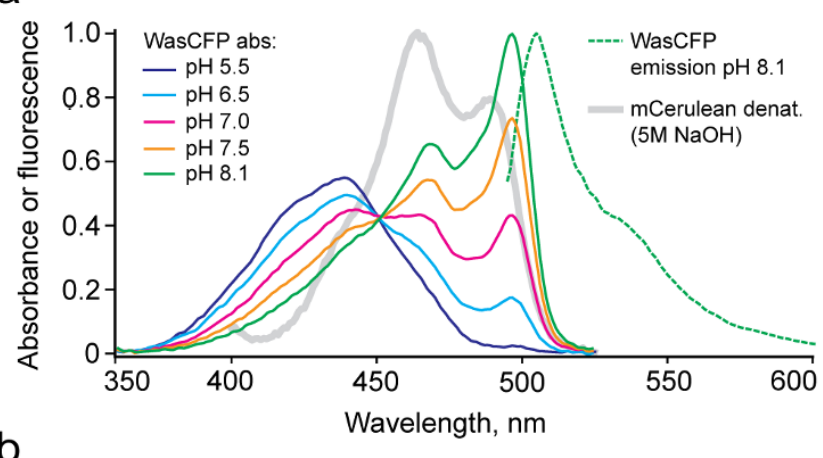

b

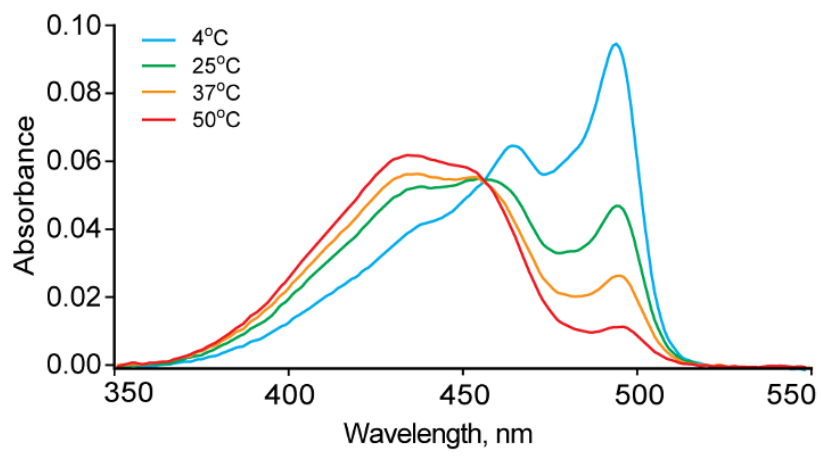

C

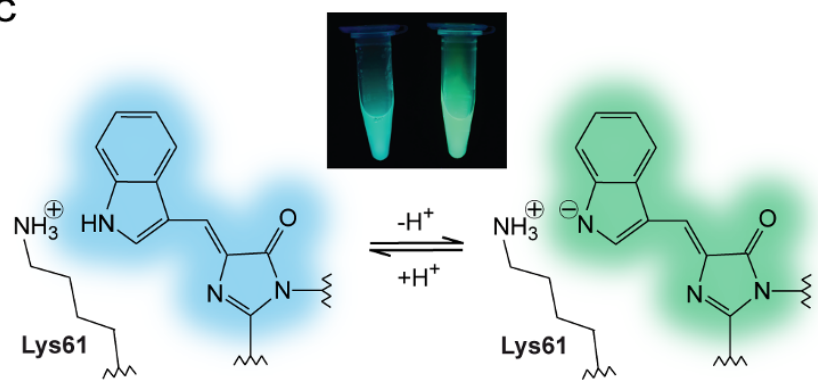

Figure $2 \mid$ Spectral properties of WasCFP and their explanation.

(a) WasCFP absorption spectra at various $\mathrm{pHs}$ (solid colored lines) at $4{ }^{\circ} \mathrm{C}$ with conversion of cyan to green-emitting forms. Dashed green lineemission at $\mathrm{pH}$ 8.1. Gray line - absorption of mCerulean denaturated in strong alkaline conditions. (b) Temperature dependence of WasCFP absorption spectra (at pH7.4). (c) A proposed explanation of the bathochromic shift in WasCFP. Neutral cyan chromophore (left) undergoes ionization upon $\mathrm{pH}$ increase. Resulting green-emitting anionic state of the chromophore (right) is stabilized by positively charged lysine61 side chain. Photo inset shows fluorescence of WasCFP solution under UV lamp at pH 5 (left) and $\mathrm{pH} 8$ (right). from the global fit upon $372 \mathrm{~nm}$ excitation since upon $467 \mathrm{~nm}$ excitation the excitation is being absorbed by the green species predominantly.

\section{Discussion}

In WasCFP, V61K appears to be a key substitution as this single mutation was sufficient to promote appearance of the green fluorescent state. Although $\mathrm{pKa}$ of a Lys side chain is 10.4 in aqueous solution, it is known that it can be as high as 12.1 within the protein ${ }^{11}$. Other mutations further shifted equilibrium from cyan to green state and improved overall folding and brightness. From the Cerulean crystal structure $^{12}$ it is known, that the side chain of amino acid in the position 207 is in tight contact with the side chain of amino acid in the position 61. Therefore, substitution L207Q likely participates in fine tuning of Lys61 conformation. Substitutions in the position 148 are well-known to influence chromophore properties ${ }^{1-4}$. We speculate that substitution of Asp148 for smaller amino acid residue Gly provides additional space near the chromophore, allowing it to adapt microenvironmental changes caused by bulky side chain of Lys61.

Does WasCFP chromophore exist in the anionic state? Chromophore ionization in fluorescent proteins is hard to observe directly. Following classical studies of GFP chromophore protonation state, we applied $\mathrm{pH}$-titrations and other indirect evidences to answer this question.

The strongest evidence for anionic nature of chromophore in WasCFP is $\mathrm{pH}$ titration data. Indeed, $\mathrm{pH}$ increase from acidic to mild basic conditions results in cyan-to-green conversion of WasCFP. This transition is highly similar to analogous $\mathrm{pH}$-dependent bathochromic shift in various green, yellow and red FPs with Tyrbased chromophores, where chromophores' phenolic oxygen deprotonation occurs ${ }^{1-3}$. Moreover, absorption spectrum of mCerulean denatured in strong alkaline conditions (5 M NaOH, mCerulean chromophore is anionic) is red-shifted similarly to WasCFP green form.

Also, green form appeared as a result of introduction of positively charged Lys, which can stabilize chromophore's negative charge. Temperature dependence of WasCFP can be explained in terms of Lys61/chromophore interaction. Taking into account a high $\mathrm{p} K_{\mathrm{a}}$, proton abstraction from the Trp-based chromophore should be a delicate process, and should depend strongly on the proximity of Lys61 side chain to the indole nitrogen. Therefore, due to higher mobility of protein backbone and side chains at higher temperatures, one can expect the green form to disappear when heating.

Two other potential explanations of the observed bathochromic shift in WasCFP can be considered. First, formation of DsRed-type acylimine is known to extend conjugated electron system of the chromophore and results in a strong red shift. An example of fluorescent protein with Trp-based acylimine-containing chromophore is
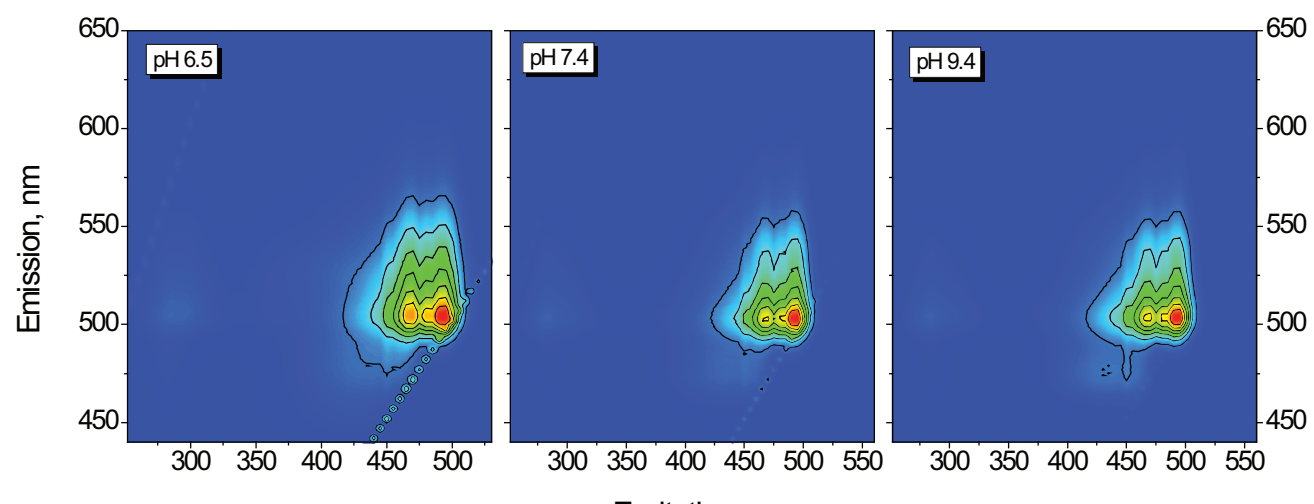

\section{Excitation, $\mathrm{nm}$}

Figure 3 Excitation-emission contour plots of WasCFP at various pH. The difference between the neighboring lines corresponds to the constant intensity difference on a linear scale. Red color corresponds to higher intensities. 

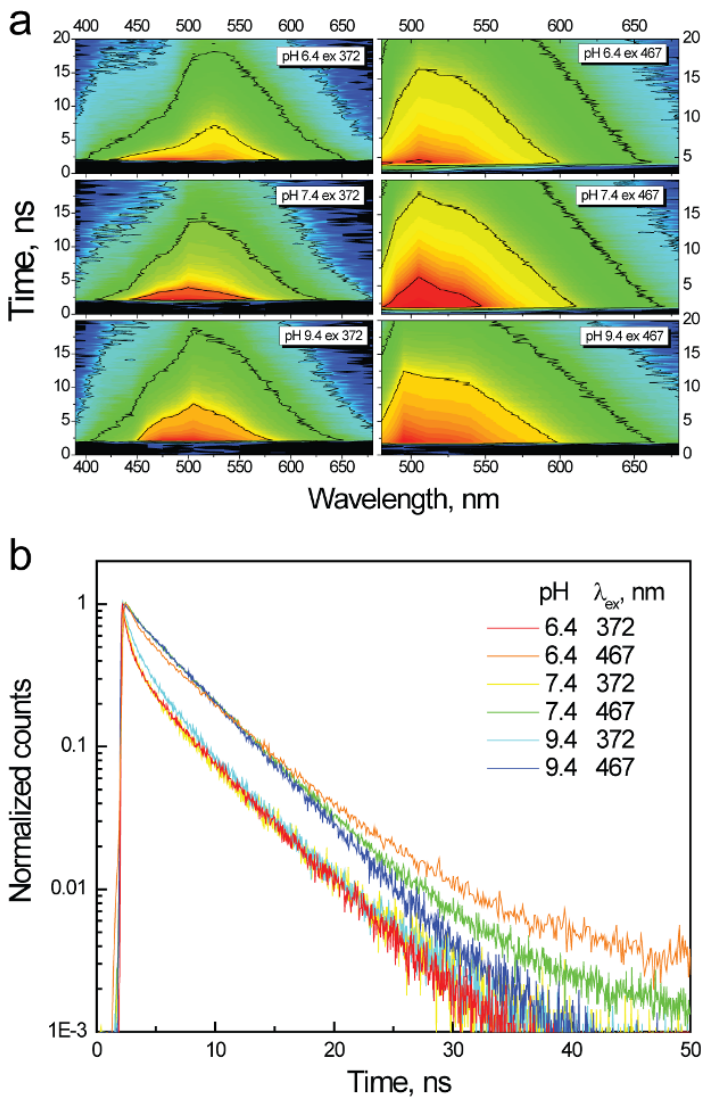

Figure 4 | Time-resolved fluorescence spectra of WasCFP. (a) Emission decay contour plots of WasCFP at various $\mathrm{pH}$. The difference between the neighboring lines corresponds to the constant intensity difference on a logarithmic scale. Red color corresponds to higher intensities.

(b) Fluorescence decay curves of WasCFP detected at $510 \mathrm{~nm}$ under $372 \mathrm{~nm}$ or $467 \mathrm{~nm}$ excitation in solutions with various $\mathrm{pH}$.

mHoneydew - a mutant of monomeric red fluorescent protein $\mathrm{mRFP}^{13}$. This protein has broad double-peaked spectra with excitation peaks at 487 and $504 \mathrm{~nm}$, and emission peaks at 537 and $562 \mathrm{~nm}$. mHoneydew shows high $\mathrm{pH}$ stability $\left(\mathrm{p} K_{\mathrm{a}}<4\right)$. All these properties are very different from that of WasCFP. Also, at denaturing protein gel-electrophoresis WasCFP did not show protein chain fragmentation (Supplementary Fig. 5), which is characteristic for proteins with DsRed-type chromophore ${ }^{14}$. Generally, formation of DsRed-type acylimine can hardly be responsible for fast and reversible $\mathrm{pH}$ - and temperature-dependent interconversions of green and cyan forms in WasCFP.

Second possibility of bathochromic shift is stacking interactions between the chromophore and an aromatic amino acid from its environment. This effect was first discovered for yellow mutants of GFP with Tyr203 as a key stacking residue ${ }^{1,15}$. Introduction of Tyr203 into ECFP resulted in a mutant named ECGFP with a red shifted spectra (absorption and emission maxima at 463 and $506 \mathrm{~nm}$, respectively $)^{16}$. In contrast to WasCFP, ECGFP was extremely $\mathrm{pH}$ stable and showed practically no changes in absorption spectrum in a $\mathrm{pH}$ range from 4 to 12 . Clear dissimilarity of ECGFP and WasCFP together with absence of aromatic residues among WasCFP-specific mutations allowed us to conclude that stacking interactions are not responsible for red shifted spectra of WasCFP.

To conclude, we presented the first evidence strongly suggesting that tryptophan-based chromophores in fluorescent proteins can exist in the anionic state. It is also the first case of deprotonation of the indole substituent in a biological system. Switching between protonated and deprotonated Trp66 in fluorescent proteins represents a new unexplored way to control their spectral properties. The main advantage for practical applications of WasCFP is its extremely high fluorescence lifetime of the green form (5.2 ns; for comparison, fluorescence lifetime of EGFP is $2.3 \mathrm{~ns}^{17}$ ). To the best of our knowledge, this is the highest value of fluorescence lifetime among GFPlike fluorescent proteins. Thus, WasCFP is probably a useful tag for fluorescence lifetime imaging microscopy (FLIM) that can be used to discriminate two or more fluorescent proteins of the same color but with different fluorescence lifetimes by lifetime unmixing. Also, high quantum yield and fluorescence lifetime of WasCFP makes it a potentially excellent donor for fluorescence resonance energy transfer (FRET). While WasCFP can probably be successfully applied as it is, further molecular evolution of WasCFP would be applied to minimize residual amounts of protein in the uncharged state and thus suppress its unwanted cyan fluorescence. High environmental sensitivity (in particular, to urea) of WasCFP suggests that even subtle changes of protein conformation result in clearly detectable changes of its spectra. This property is desirable for construction of genetically encoded sensors, in which conformational changes of sensitive domains should induce conformational changes of a fluorescent protein and corresponding detectable modulation of its fluorescence. We expect that our finding will open new perspectives for development of sensors and photoconvertible fluorescent proteins with Trp-based green or red chromophores.

\section{Methods}

CFP chromophore synthesis. CFP chromophore analog ((5Z)-5-(1H-indol-3ylmethylidene)-2,3-dimethyl-3,5-dihydro-4H-imidazol-4-one, Supplementary Fig. 1a) was synthesized as described ${ }^{8}$

Mutagenesis. The set of positions potentially providing sufficient proximity of Lys and Arg side chains to the chromophore was selected on the basis of virtual mutagenesis of Cerulean crystal structure (PDB: 2WSO) in PyMol package (The PyMOL Molecular Graphics System, Version 0.99, Schrödinger, LLC). We selected positions for which a contact between Lys or Arg side chains and chromophore was observed at least in one of the conformations proposed by PyMol.

Synthetic DNA oligonucleotides for mutagenesis were purchased from Evrogen. PCRs were carried out using PTC-100 thermal cycler (MJ Research). Purification of PCR products and products of restriction digests was performed by gel electrophoresis and extraction using the QIAquick gel extraction kit (Qiagen). Plasmid DNA was purified using the QIAprep Spin Miniprep kit (Qiagen). Site-directed mutagenesis was performed by overlap-extension $\mathrm{PCR}^{18}$. Random mutagenesis was performed using the Diversity PCR Random Mutagenesis kit (Clontech). For bacterial expression, a fluorescent protein gene was cloned into the $\mathrm{PQE}-30$ vector (Qiagen) using BamHI and HindIII restriction sites. Restriction endonucleases were purchased from New England Biolabs.

Selection of mutants was based on visual screening of $E$. coli colonies expressing mutant proteins under Olympus SZX12 research stereo zoom microscope.

Purification and characterization of fluorescent proteins in vitro. Fluorescent proteins were expressed in E. coli XL1 Blue strain (Invitrogen) in the LB medium, centrifuged, sonicated in PBS ( $\mathrm{pH}$ 7.4), then purified using Talon metal-affinity resin (Clontech). Cary 100 UV/VIS Spectrophotometer and Varian Cary Eclipse Fluorescence spectrophotometer were used to measure absorption and excitationemission spectra. Quantum yields were determined by direct comparison with EGFP and mCerulean.

Extinction coefficients of WasCFP cyan and green forms were determined as follows. Synthetic CFP chromophore in basic dimethylformamide possessed doublepeaked absorption curve with two close maxima at 460 and $473 \mathrm{~nm}$ and extinction coefficient $46000 \mathrm{M}^{-1} \mathrm{~cm}^{-1}$ at both wavelengths (Supplementary Fig. 1d). This value was used to calculate amount of mature protein in a sample of alkali-denatured WasCFP (absorption spectrum with two maxima at 464 and $487 \mathrm{~nm}$, Supplementary Fig. 1c). Native WasCFP is a mixture of cyan and green forms in equilibrium. We calculated extinction coefficients of both forms using spectra of WasCFP at conditions favored strong dominance of either cyan or green form ( $\mathrm{pH} 5.5$ and 8.1 at $4{ }^{\circ} \mathrm{C}$ ).

To measure the $\mathrm{pH}$ dependence of spectra, we used buffers in the $\mathrm{pH}$ range from 5 to 11. An aliquot of purified protein was diluted in the corresponding buffer solution. Spectra were measured at room temperature $\left(25^{\circ} \mathrm{C}\right)$ or at $4^{\circ} \mathrm{C}$ after $15 \mathrm{~min}$ of incubation in buffer. In each sample, actual final $\mathrm{pH}$ was measured using a microelectrode (Sartorius).

To measure the temperature dependence of spectra we used Cary 100 UV/VIS spectrophotometer equipped with Cary temperature controller. Spectra were measured after $5 \mathrm{~min}$ of incubation at corresponding temperature.

To measure the urea dependence of spectra, a set of phosphate buffers with urea concentration from $0.125 \mathrm{M}$ to $10 \mathrm{M}$ was prepared. After protein addition $\mathrm{pH}$ values 
of all solutions differed slightly in the range 7.9-8.2. After 15 min incubation at room temperature $\left(25^{\circ} \mathrm{C}\right)$, the absorbance was measured.

Fluorescence lifetimes were measured using an Edinburgh Instruments timecorrelated single photon counting (TCSPC) system. In these measurements, picosecond excitation pulses diode lasers (Picoquant) emitting at $372 \mathrm{~nm}$ or $467 \mathrm{~nm}$ was used as an excitation light source. The detection system consisted of a high speed MicroChannel Plate PhotoMultiplier Tube (MCP-PMT, Hamamatsu R3809U-50) and TCSPC electronics. The time resolution of the system was $30 \mathrm{ps}$ after deconvolution with an IRF signal. Global analysis of the kinetic data was performed using the publicly available package Glotaran 1.2 (http://www.glotaran.org) ${ }^{19}$.

1. Chudakov, D. M., Matz, M. V., Lukyanov, S. A. \& Lukyanov, K. A. Fluorescent proteins and their applications in imaging living cells and tissues. Physiol. Rev. 90, 1103-1163 (2010).

2. Piatkevich, K. D., Malashkevich, V. N., Almo, S. C. \& Verkhusha, V. V. Engineering ESPT pathways based on structural analysis of LSSmKate red fluorescent proteins with large Stokes shift. J. Am. Chem. Soc. 132, 10762-10770 (2010).

3. Tsien, R. Y. The green fluorescent protein. Annu. Rev. Biochem. 67, 509-544 (1998)

4. Rizzo, M. A., Springer, G. H., Granada, B. \& Piston, D. W. An improved cyan fluorescent protein variant useful for FRET. Nat. Biotech. 22, 445-449 (2004).

5. Markwardt, M. L. et al. An Improved Cerulean fluorescent protein with enhanced brightness and reduced reversible photoswitching. PLoS One 6, e17896 (2011).

6. Goedhart, J. et al. Bright cyan fluorescent protein variants identified by fluorescence lifetime screening. Nat. Methods 7, 137-139 (2010)

7. Goedhart, J. et al. Structure-guided evolution of cyan fluorescent proteins towards a quantum yield of 93\%. Nat. Commun. 3, 751 (2012).

8. Kojima, S. et al. Fluorescent properties of model chromophores of tyrosine-66 substituted mutants of Aequorea green fluorescent protein (GFP). Tetrah. Lett. 39, 5239-5242 (1998)

9. Ward, W. W. Biochemical and physical properties of green fluorescent protein. In: Green Fluorescent Protein: properties, applications, and protocols. $\left(2^{\text {nd }}\right.$ ed., Chalife, S. \& Kain, S., Eds.), John Wiley \& Sons, New Jersey, pp. 39-65 (2006).

10. Verkhusha, V. V. et al. High stability of Discosoma DsRed as compared to Aequorea EGFP. Biochemistry 42, 7879-7884 (2003).

11. Kesvatera, T., Jönsson, B., Thulin, E. \& Linse, S. Measurement and modelling of sequence-specific pKa values of lysine residues in calbindin D9k. J. Mol. Biol. 259, 828-839 (1996).

12. Lelimousin, M. et al. Intrinsic dynamics in ECFP and Cerulean control fluorescence quantum yield. Biochemistry 48, 10038-10046 (2009).

13. Shaner, N. C. et al. Improved monomeric red, orange and yellow fluorescent proteins derived from Discosoma sp. red fluorescent protein. Nat. Biotechnol. 22, $1567-1572(2004)$
14. Gross, L. A., Baird, G. S., Hoffman, R. C., Baldridge, K. K. \& Tsien, R. Y. The structure of the chromophore within DsRed, a red fluorescent protein from coral. Proc Natl Acad Sci USA 97, 11990-11995 (2000).

15. Ormö, M. et al. Crystal structure of the Aequorea victoria green fluorescent protein. Science 273, 1392-1395 (1996).

16. Sawano, A. \& Miyawaki, A. Directed evolution of green fluorescent protein by a new versatile PCR strategy for site-directed and semi-random mutagenesis. Nucleic Acids Res. 28, E78 (2000).

17. Ito, T., Oshita, S., Nakabayashi, T., Sun, F., Kinjo, M. \& Ohta, N. Fluorescence lifetime images of green fluorescent protein in HeLa cells during TNF-alpha induced apoptosis. Photochem. Photobiol. Sci. 8, 763-767 (2009).

18. Higuchi, R., Krummel, B. \& Saiki, R. K. A general method of in vitro preparation and specific mutagenesis of DNA fragments: study of protein and DNA interactions. Nucleic Acids Res. 16, 7351-7367 (1988).

19. Snellenburg, J. J., Laptenok, S. P., Seger, R., Mullen, K. M. \& van Stokkum, I. H. M. Glotaran: A Java-Based Graphical User Interface for the R Package TIMP. J. Stat. Soft. 49 (3), 1-23 (2012).

\section{Acknowledgments}

This work was supported by the Ministry of Education and Science of the Russian Federation (project No. 11.G34.31.0017), by Molecular and Cell Biology program of the Russian Academy of Sciences, by Russian Foundation for Basic Research (grant No. 12-04 33125) and by the U.S. National Science Foundation (CHE-0809179 and 1213047 to KMS).

\section{Author contributions}

KSS performed mutagenesis and obtained spectral data. IVY synthesized and studied CFP chromophore. KMS performed 3D and time-resolved spectral measurements. ASM together with SAL and KAL designed and supervised the project. ASM, KAL, KSS and KMS wrote the paper, and all authors reviewed the manuscript.

\section{Additional information}

Supplementary information accompanies this paper at http://www.nature.com/ scientificreports

Competing financial interests: The authors declare no competing financial interests.

License: This work is licensed under a Creative Commons

Attribution-NonCommercial-NoDerivative Works 3.0 Unported License. To view a copy of this license, visit http://creativecommons.org/licenses/by-nc-nd/3.0/

How to cite this article: Sarkisyan, K.S. et al. Tryptophan-based chromophore in fluorescent proteins can be anionic. Sci. Rep. 2, 608; DOI:10.1038/srep00608 (2012) 